\title{
Filigrane
}

Écoutes psychothérapiques

\section{De l'orientation professionnelle à la pulsion invocante : « l’appel d'appel »}

\section{Frédéric Vinot}

Volume 17, numéro 1, printemps 2008

L’avenir du clinicien I

URI : https://id.erudit.org/iderudit/018795ar

DOI : https://doi.org/10.7202/018795ar

Aller au sommaire du numéro

Éditeur(s)

Revue Santé mentale au Québec

ISSN

1192-1412 (imprimé)

1911-4656 (numérique)

Découvrir la revue

Citer cet article

Vinot, F. (2008). De l'orientation professionnelle à la pulsion invocante :

«l'appel d'appel ». Filigrane, 17(1), 155-164. https://doi.org/10.7202/018795ar

\section{Résumé de l'article}

Dans cet article, l'auteur propose une approche de la pratique de l'orientation professionnelle à partir de la psychologie clinique se soutenant du référentiel psychanalytique. La question est abordée au travers d'un type de demande rencontré chez certains demandeurs d'emploi : la recherche de vocation. À être traduite comme " appel d'appel ", cette position subjective peut être entendue métapsychologiquement au travers du circuit de la pulsion invocante, proposée par J. Lacan. Un cas clinique montre comment loin de forcément développer la vocation (comme le voudrait le discours social), le clinicien peut au contraire favoriser une nouvelle position qui consiste plutôt à renoncer à jouir d'un certain versant de la voix : renoncer à se savoir appelé par l'Autre. 


\title{
De l'orientation professionnelle à la pulsion invocante: "l'appel d'appel»
}

\author{
frédéric vinot
}

\begin{abstract}
Dans cet article, l'auteur propose une approche de la pratique de l'orientation professionnelle à partir de la psychologie clinique se soutenant du référentiel psychanalytique. La question est abordée au travers d'un type de demande rencontré chez certains demandeurs d'emploi : la recherche de vocation. À être traduite comme «appel d'appel», cette position subjective peut être entendue métapsychologiquement au travers du circuit de la pulsion invocante, proposée par J. Lacan. Un cas clinique montre comment loin de forcément développer la vocation (comme le voudrait le discours social), le clinicien peut au contraire favoriser une nouvelle position qui consiste plutôt à renoncer à jouir d'un certain versant de la voix : renoncer à se savoir appelé par l'Autre.
\end{abstract}

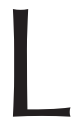

a question de l'orientation professionnelle a commencé à se poser en France vers 1900, sous la forme du choix du métier à la sortie de l'école. 1900, c'est aussi l'année de parution de L'interprétation des rêves. Mais il faut constater que bien que contemporaines, les recherches sur l'aide à l'orientation professionnelle et les recherches psychanalytiques se sont jusqu'à présent quasiment ignorées. L'ouvrage exhaustif de M. Huteau et J Guichard, Psychologie de l'orientation (2001) en témoigne: le dialogue pratique et épistémologique entre orientation et psychanalyse ne va pas de soi. Or (doit-on y déceler un effet des figures actuelles du malaise dans la culture?), les psychologues cliniciens sont de plus en plus sollicités pour intervenir dans les champs de l'orientation et de l'insertion professionnelle. Très rapidement certaines questions se posent pour ceux qui relèvent ce défi: comment exercer dans ce nouveau champ en maintenant pertinente et vivante la référence métapsychologique ? Quels aménagements cliniques, quelles «variantes» de la cure type, sont-ils nécessaires? Et en retour quels enseignements en tirer pour la psychanalyse? Mon propos ne sera pas de répondre à l'ensemble de ces questions, mais plutôt de tirer de nouvelles conséquences d'un des postulats freudiens les plus fermes. Freud, dans son œuvre, a sans cesse pensé l'établissement et l'insertion dans la Culture à partir de la dynamique pulsionnelle. C'est précisément une variante de cette dynamique pulsionnelle dans ses liens avec l'insertion que je m'attacherai à déployer. Nous verrons ainsi comment une position subjective particulière, l'appel d'appel, peut être entendue à partir de la proposition lacanienne d'une pulsion invocante.

Travaillant il y a quelques années dans un centre d'orientation, j'ai reçu une jeune femme de 28 ans que j'appellerai $\mathrm{M}^{\mathrm{Hle}}$ M.. Originaire du Nord de la France, 
elle obtient une maîtrise d'italien, commence à travailler dans l'animation, mène une action de jumelage municipal, puis travaille dans la restauration à Paris. La profonde insatisfaction que lui procure cette dernière activité sera à l'origine de son départ, et de son arrivée à N..., où l'ANPE me l'oriente dans le cadre d'une mesure d'accompagnement à la « définition de projet professionnel».

D'entrée, elle me dit chercher «sa voie». Or, toute possibilité d'emploi, de formation est accueillie par un «pas assez». Il manque toujours quelque chose: rien n'est à la hauteur de cette voie qu'elle ne peut imaginer, aucune possibilité n'est à même de remplir autant ses attentes que cette voie dont elle ignore tout sauf qu'elle a la certitude qu'elle existe. Peu à peu, en même temps qu'elle remet en question la pertinence de l'accompagnement, elle fait état d'une accentuation de sa désorientation : «Je suis dans la brume, je suis perdue» puis «je panique». Son angoisse est intense, à tel point que je me mets moi-même à chercher ce qu'elle pourrait effectivement faire, en quoi pourrait consister ce métier idéal qui doit bien exister quelque part, bref ce pour quoi elle est faite...

Le rapatriement dans le site de l'analyse me permet de me dégager de cette position en miroir fort inconfortable. Je suis alors en mesure de lui faire part de cette attente intense qu'elle évoque, qui n'est jamais assouvie. Ce à quoi elle répond: «De toute façon je sais bien ce que je veux faire, c'est écrire. » D'où lui vient cette idée? Quelqu'un écrit-il dans ses proches ? Sa mère: «Ma mère, elle écrit. Elle garde, moi je jetais ce que j'écrivais. De toute façon je sais ce que je veux faire, mais je n'ose pas. » J'arrête l'entretien là-dessus.

Dès lors, et sans anticipation possible de ma part, elle se rendra quelques jours plus tard à un entretien pour une formation dans une position subjective complètement différente de celle qu'elle occupait auparavant (qui était celle du «pas assez»). Cette fois-ci, me dira-t-elle plus tard, elle n'attendait «rien». Et c'est à ce moment, en écoutant parler la responsable de la formation, que lui viennent des idées : «Finalement, ça m'a intéressé, j’ai même eu quelques idées pendant l'entretien. »

\section{L'appel d'appel : description phénoménologique}

Dans le cadre de demande d'orientation, il apparaît quelques fois un discours dans lequel le sujet fait état d'une attente particulière. La demande d'aide quant au choix professionnel contient un point central : la personne vient chercher l'activité «qui fait tilt», ou encore comme le disent certains «mon truc» ou «ma voie». Le choix de cette activité apparaîtrait alors sans incertitude possible, dans l'évidence d'un coup de foudre. L'incertitude, la prise de risque inhérente à tout choix n'est plus supportable. La déception, l'ennui, voire la tromperie ont été au rendez-vous des précédents emplois (quand il y en a eu) et la demande porte en elle une exigence à ne plus les rencontrer à nouveau. Comme si le métier (à différencier de l'emploi) était en lui-même responsable des rapports que le sujet entretient avec l'Autre. Comme il est dit parfois explicitement, il s'agit ici d'une véritable recherche de vocation professionnelle. Une instance psychique souhaite, demande 
puis rapidement exige d'être appelée par le biais du praticien (vocation vient du latin vocare: appeler). Il s'agit d'un appel à être appelé. L'expression «sujet de l'appel d'appel», sur laquelle nous reviendrons, trouve ici son origine. Nous le verrons, ce que la prise transférentielle permet de supposer, c'est que plus la demande est forte vis-à-vis du praticien, plus on est en droit d'y entendre une injonction interne à laquelle le sujet ne peut que se plier: «Tu dois savoir quelle est ta voie !» Retenons donc cette idée d'une voix d'injonction. Or, plus le sujet appelle à être appelé et plus il se trouve perdu, désorienté, dans l'incapacité de se sentir attiré vers, appelé vers.

En effet, en parallèle de cet appel une inhibition au choix se manifeste, et c'est souvent ce qui motive l'occasion de la rencontre. Le métier recherché est très souvent décrit comme exempt de tout prix à payer : c'est un travail qui ne mobilise plus d'angoisse, qui rapporte gros, qui satisfait à toutes les conditions imaginaires. En fait c'est un emploi qui souhaiterait faire l'économie de la castration. La recherche de vocation idéalise ainsi le rapport au travail, en rejetant sa part de souffrance, que rappelle pourtant la langue française dans son étymologie. De fait, lorsqu'une opportunité professionnelle se présente (offre d'emploi, proposition ou formation) celle-ci est rejetée comme n'étant pas à la hauteur de cette vocation imaginaire non déterminée...

Avec ce «tu dois savoir quelle est ta voie!», l'élaboration du projet se trouve donc entièrement soumise au champ d'un certain savoir. Mais de quel ordre est ce savoir? Il nous faut remarquer que cette injonction particulière prête à réflexion car on peut tout à fait y repérer le mot d'ordre d'épanouissement propre à notre idéal contemporain. En écho à cette injonction de savoir, ces dernières décennies ont vu se développer une approche de l'aide au choix professionnel centrée sur un modèle éducatif souvent soumis à des références socio-cognitives dans lesquelles toute division est ignorée: le sujet y est donc compris comme indivis et, sur le projet, règne tout aussi bien l'intentionnalité consciente que le fantasme d'un savoir qui dissiperait définitivement ce qu'il en est de l'énigme du désir. C'est donc un savoir au sens positiviste du terme. Il n'est pas à identifier au savoir inconscient issu de l'expérience psychanalytique, mais bien plutôt à son acception traditionnelle, extensive à la réflexion d'un sujet connaissant — c'est-à-dire relevant des processus moïques encouragés par un idéal narcissique de maîtrise (Vandermersch et Chemama, 1998, 379). Ainsi, au «je dois savoir» du demandeur d'emploi, le conseiller en orientation ou en insertion répond parfois « oui, tu dois savoir». Inutile ici de préciser les effets ravageurs de cette relation en miroir lorsque aucun lieu de parole ne vient atténuer la prédominance de ces enjeux imaginaires. En cela, le «tu dois savoir» devient la grimace ricanante du «tu peux savoir» (silicet).

\section{L'appel d'appel : déploiements métapsychologiques}

Alors qu'est-ce qu'une clinique d'orientation psychanalytique peut apporter à ceci ? En quoi pourrait-elle contribuer à repérer les enjeux inconscients de cette 
exigence de vocation? Et comment peut-elle objecter à cette tyrannie du savoir, aussi bien individuelle qu'institutionnelle, voire sociale?

Accueillir cette exigence de vocation professionnelle sur un mode uniquement éducatif ne permet guère de repérer à quelle instance psychique référer la férocité de cette demande. Il faut en situer la dimension transférentielle pour que cette exigence de révélation de vocation, adressée au clinicien, soit entendue en son versant inconscient, c'est-à-dire comme la terrible vocation d'un surmoi implacable. $\mathbf{M}^{\text {lle }}$ M. adresse bien son insatisfaction grandissante en direction du praticien, lequel présentifie ce lieu de l'Autre: tout ce qui ne présente pas les caractéristiques d'une vocation, à savoir la fin du doute et de l'incertitude, est rejeté. La remise en question de ses recherches et de l'utilité de l'accompagnement vont grandissants ${ }^{1}$ : ça ne vaut pas la peine d'être venue de si loin, d'avoir déjà tant payé, si c'est pour reprendre un emploi qui ne soit pas à la hauteur de cette attente ! À partir de là, nous pouvons déployer cet impératif qui règne sans partage sur la demande. Ce déploiement peut se faire sur deux versants.

Le premier versant: la dimension d'injonction interne révélée et permise par le transfert permet de comprendre le « il doit y avoir une voie pour moi! » comme « il y a une voie pour toi!». Autrement dit, au travers de l'injonction à laquelle $\mathrm{M}^{\text {lle }} \mathrm{M}$. soumet l'autre, on peut entendre celle à laquelle l'Autre la soumet. Le sujet se trouve donc soumis ici à cet impératif surmoïque (ce que nous nommons injonction interne). Or, le surmoi en appelle à la jouissance (Lacan, 1975, 10) ${ }^{2}$. Cette indication peut alors nous permettre de comprendre cette inhibition dont fait état le sujet de l'appel d'appel. Il y a une dimension indéniable de jouissance pour qui se trouve dans la recherche éperdue d'un métier idéal. Mais avec quel objet cette jouissance se trouve-t-elle être en lien?

Le second versant nous permet d'avancer sur cette question, car «il y a une voie pour toi ! p pourrait être entendu comme un tout aussi féroce «il y a une voix pour toi !». En effet, Freud dès 1914 avait déjà repéré cette origine vocale de l'instance critique par le biais de ce qu'il identifiait alors comme idéal du moi :

«Ce qui avait incité le sujet à former l'idéal du moi dont la garde est remise à la conscience morale, c'était justement l'influence des parents telle qu'elle se transmet par leur voix » (Freud, 1989, 100)

Plus tard, dans «Le moi et le ça » Freud, conserve au surmoi son essence vocale et donne à l'entendu une prévalence qui le pousse à ajouter à son schéma de l'appareil animique la fameuse «calotte acoustique» (Freud, 1991, 269). Mais chez Freud, la voix (y compris et surtout dans sa «fonction psychique») garde toujours un lien avec l'entendu, c'est-à-dire qu'elle continue à relever de la dimension sonore (Lecourt, 1992) et, en tant que telle, ne peut accéder à un statut pulsionnel. C'est avec Lacan que l'on va pouvoir penser davantage les liens entre jouissance et voix... 
La proposition lacanienne d'une «pulsion invocante» $(1973,182)$ nous permet en effet d'envisager une autre facette de cette exigence de vocation que profère le sujet de l'appel d'appel. L'avancée de Lacan est de référer cette pulsion invocante non plus à la voix de la phoniatrie, à la voix sonore, mais bien plutôt à la dynamique inconsciente de l'appel et du se faire entendre. Au niveau des représentants langagiers, elle peut par exemple se décliner entre un «appeler», «être appelé », «se faire appelé» (à l'occasion de tous les noms...), ou aussi bien dans l'«entendre », «être entendu », ou «se faire entendre». On comprend dès lors que si ce à quoi certains demandeurs d'emplois appellent de toutes leurs forces, c'est précisément à être appelés, le circuit de la pulsion invocante se trouve être profondément en cause dans cette position subjective. Comment penser ce circuit?

Freud, et à sa suite Lacan, n'ont eu de cesse de rappeler que le devenir pulsionnel devait se penser en termes dynamiques. L'expression «circuit pulsionnel» (Lacan, 1973, 163) rend ainsi compte des trois temps nécessaires à la pulsion pour (ne pas) atteindre sa satisfaction. Dans «Pulsions et destins des pulsions » Freud proposait d'analyser l'activité pulsionnelle à partir (entre autre) du couple d'opposés pulsionnels regarder/se montrer. Les trois temps de ce qui sera plus tard appelé «pulsion scopique» s'établissent en terme de retournement-renversement, indiquant déjà son caractère circulaire. Voilà comment Freud décrit ces trois temps :

«a) Regarder, comme activité dirigé sur un objet étranger.

b) Abandon de l'objet, retournement de la pulsion de regarder sur une partie du corps propre; en même temps : renversement en passivité et instauration d'un nouveau but: être regardé.

c) Introduction d'un nouveau sujet auquel on se montre pour être regardé par lui.» (1915-1968, 28-29)

C'est avec ce troisième temps (celui que Lacan nommera le «se faire ») que la pulsion «atteint sa satisfaction sans atteindre son but» car «son but n'est point autre chose que ce retour en circuit» (Lacan, 1973, 163). Arrivé à ce troisième temps, la pulsion peut donc reprendre son circuit et relancer la quête de l'objet perdu. On peut donc se poser la question: dans le cas du sujet de l'appel d'appel, qu'en est-il de cette relance pulsionnelle?

Pour y répondre, il nous faut préalablement proposer une transposition de ces trois temps à la pulsion invocante ${ }^{3}$. Reprenant la formulation freudienne, les trois temps de la pulsion invocante pourraient donc être pensés de cette façon:

a) Etre appelé : aux origines de son existence, l'infans pousse un cri sous l'effet d'une pure tension endogène, impossible à réguler du fait de sa prématurité. Peu importe que ce cri soit le premier ou un autre. Ce qui importe c'est qu'il soit une manifestation vocale liée à son état de prématurité. Ce cri n'est pas d'abord appel. Il ne pourra le devenir qu'après la réponse de l'Autre. Il revient à l'Autre d'attribuer une signification à ce cri pur, autrement dit de l'interpréter. Cette 
interprétation, si elle se réfère au registre du besoin (le cri comme signe de la faim, de la soif, etc.) est également marquée par le désir de l'Autre: "Que veux-tu que je te veuille?» L'Autre se positionne donc en récepteur et crée de ce fait, après coup, un émetteur. En accusant réception de ce cri, l'Autre émet l'hypothèse que ce cri veut dire quelque chose: c'est le temps de l'appel de l'Autre. D'où le fait que le premier temps de la pulsion humanisante soit «être appelé» et non pas «appeler». Nous sommes au plus proche de ce que Freud appelait «expérience de satisfaction» (Freud, 1996, 336).

b) Appeler: ce n'est seulement qu'après ce premier temps que le cri pur deviendra cri pour, et donc appel. L'intervention de l'Autre introduit l'infans au registre de la signification - lui faisant dès lors perdre pour toujours l'immédiateté du rapport à la voix comme objet (la voix comme objet $a$ ). Dès lors, plus jamais, le sujet ne pourra retrouver cette dimension du cri pur. L'entrée dans la parole vient voiler la voix, et le cri pur cède la place à l'appel. C'est ici le temps de l'appel à l'Autre, Autre qui est sommé de se manifester à nouveau comme la première fois... Mais cela est-il possible?

c) Se faire appeler: si le troisième temps peut ne pas être une redite du premier, c'est qu'entre temps la dynamique désirante s'est mise en place. Il devient alors impossible au sujet de retrouver l'instant mythique de l'appel de l'Autre sans se situer lui-même comme aliéné dans ce désir. On perçoit ici la modification radicale entre le premier et le troisième temps: le sujet n'est plus créé par le désir, l'appel de l'Autre, il y est maintenant aliéné. Dès lors le «se faire appelé» nous rapproche de l'axiome lacanien selon lequel le désir de l'homme est le désir de l'Autre (Lacan, 1966, 343). En appelant, le sujet découvre qu'il ne peut que se faire appelé, car condamné à compter avec la fonction inconsciente du désir de l'Autre. Le «se faire appelé » serait un des modes de compréhension de la mise en mouvement du désir. Il doit être conçu comme une véritable passivation ${ }^{4}$ de la pulsion invocante, marquant l'avènement du sujet comme assujetti. Dans ce troisième temps, le sujet n'a plus besoin d'attendre la certitude de la révélation de l'appel (dans laquelle l'exigence de vocation est entièrement soumise au savoir conscient), mais témoigne plutôt d'un mouvement dont il ne connaît pas précisément la direction finale bref, un mouvement qui redonne ses droits au savoir inconscient. Tout comme dans le circuit pulsionnel, l'important n'est pas l'objet mais le mouvement lui-même...

Avec cette dynamique en tête, il nous semble que l'appel d'appel peut être compris comme une sorte de dysfonctionnement du circuit de la pulsion invocante. Nous proposons ainsi d'entendre cette position d'appel d'appel comme une oscillation suspendue entre le premier et le second temps de la pulsion invocante, une répétition binaire (et non ternaire) entre être appelé et appeler. Comme en témoigne $\mathrm{M}^{\mathrm{ll}} \mathrm{M}$. (pendant le premier temps de l'accompagnement), le sujet se trouve éperdument accroché à un éventuel signe de l'Autre, qui serait l'apparition soudaine de sa vocation, accomplie dans le savoir moïque. Il s'agirait donc d'une tentative de retrouver le premier temps pulsionnel sans en passer par le troisième. Ne pouvant accéder au troisième temps, la relance de la pulsion (quête désirante) 
ne peut avoir lieu. Il n'est pas anodin que l'angoisse de $\mathbf{M}^{\text {lle }} \mathrm{M}$ soit ici à son apogée: sa désorientation va en s'accentuant elle parle de «brume», «d'impression être perdue», puis «de panique». Métapsychologiquement, cette angoisse doit être entendue comme risque de la présence de l'objet pulsionnel, c'est-à-dire manque du manque. Notre hypothèse est donc que l'angoisse dans l'appel d'appel est plus à relier à la certitude qu'il y a une voie/x qu'au fait de ne pas en trouver. Cependant, l'angoisse peut aussi être prise comme la passe nécessaire signalant la confrontation du sujet à la castration puisque c'est de cela qu'il s'agit. Nous reprenons ici à notre compte la formule de Lacan $(2004,190)$ selon qui :

«l'objet perdu, c'est ce à quoi nous avons à faire d'une part dans le désir, d'autre part dans l'angoisse. Nous y avons à faire dans l'angoisse à un moment logiquement antérieur au moment où nous avons à y faire dans le désir.»

C'est précisément de ce passage réussi au troisième temps dont va témoigner $\mathrm{M}^{\text {lle }} \mathrm{M}$. lorsqu'elle dit qu'elle n'attendait rien de la rencontre avec la formatrice : la fonction prédominante jusque là accordée au savoir moïque et tyrannique semble tout à coup être barrée par le passage à un autre temps pulsionnel. L'apparition d'idées imprévues («finalement, ça m'a intéressé, j'ai même eu quelques idées pendant l'entretien ») indique quant à elle l'effet hautement symbolique du se faire appelé (relance du circuit pulsionnel). Ce passage du «pas assez» (qui relève du «trop plein » de la vocation surmoïque) à ce dessaisissement de l'attente $\mathrm{du}$ « rien » vient signer une confrontation réussie à la castration. Notons enfin que $M^{\text {lle }}$ M. indique bien que l'apparition de ces idées se fait «à l'écoute» de la responsable de formation. Tout se passe comme si elle avait pu être capacité d'entendre là une voix inouïe (ouvrant à la nouveauté), différente de la voix surmoïque, dont le message d'injonction est toujours identique à lui-même (Didier-Weill, 1995).

Mais comment le clinicien pourrait-il permettre cette relance pulsionnelle, permettre au sujet de ne plus se situer uniquement dans l'appel d'appel ? En termes freudiens, comment opérer à la mise en sourdine de la voix surmoïque appelant au savoir?

\section{Une proposition éthique et technique : l'ombilic du projet}

Dans la mythologie grecque, on connaît le chant, l'appel, mortifère des sirènes. Ce chant ne se résume pas complètement à la voix, puisque par son intermédiaire, les sirènes promettent le savoir absolu. Lisons L'odyssée:

«Allons viens Ulysse [...] Arrête ton vaisseau pour écouter notre voix. Jamais nul encore ne vint par ici sur un vaisseau noir, sans avoir entendu la voix aux doux sons qui sort de nos lèvres ; on s'en va charmé et plus savant; car nous savons tout ce que 
dans la vaste Troade souffrirent Argiens et Troyens par la volonté des dieux, et nous savons aussi tout ce qui arrive sur la terre nourricière (Homère, 1965, 179).

Cette étrange relation entre voix et savoir se retrouve également dans l'hallucination psychotique. Ce que dit l'halluciné, c'est que les voix savent, qu'il n'y a pour lui aucune moyen de remettre en doute leur verdict, d'où la certitude qui caractérise son propos. Le sujet de l'appel d'appel quant à lui serait celui qui, sans pour autant être psychotique, cède aux voix des sirènes. Il serait celui qui n'arrive pas à se rendre sourd à la promesse de savoir qui nourrit son inhibition mortifère et ne peut accéder au troisième temps de la pulsion.

Il y a donc un fil de jouissance qui relie la voix mortifère au savoir, et c'est sur ce fil-là que le clinicien peut intervenir. Il y va d'une question éthique : le maintien d'une zone de non-savoir au sein même de l'élaboration du projet, véritable «ombilic du projet» à l'instar de «l'ombilic du rêve» dont parle Freud dans L'interprétation des rêves ${ }^{5}$. Il s'agit du maintien, par le clinicien, d'un point inaccessible au savoir moïque, mais indispensable à la mise en mouvement du sujet. Selon notre hypothèse, c'est de ce point d'ombilic que la voix tyrannique peut trouver à être pacifiée, voire redevenir inaudible. On voit le pas de côté proposé par la psychanalyse: alors que toutes les techniques actuelles visent à colmater, à boucher (voire forclore) ce trou dans le savoir, la possibilité pour le praticien d'accepter et d'endurer son existence même, peut éventuellement permettre une relance du circuit invoquant chez le sujet.

Chez $\mathrm{M}^{\text {lle }} \mathrm{M}$., on a indiqué ce qui manifeste ce passage: l'apparition d'idées nouvelles à l'écoute de la responsable de formation peut être comprise comme un effet de l'efficacité de la fonction d'ombilic. Mais l'effet ne doit pas être confondu avec la cause : si celui-ci se joue au niveau de l'insertion sociale, celle-là doit être située dans le transfert. Après-coup, il nous semble que cet ombilic du projet s'est ancré dans le double décalage transférentiel décrit dans la séquence clinique initialement évoquée :

- d'une part, le clinicien trouve les moyens symboliques de se dégager de l'emprise de la voix à laquelle les manifestations transférentielles le soumettaient (Vinot, 2007). En ce sens, l'écoute clinique n'est plus guidée par la pensée parasite «il y a une voie pour elle !».

- d'autre part, l'apparition soudaine d'une autre scène sur laquelle la relation mère-fille («elle garde, moi je jetais ce que j'écrivais») se place, grâce à l'équivocité langagière, sous le signifiant de la pulsion invocante («ma mère elle écrit» — elle est cri). À ce titre, il n'est pas impossible que la voix tyrannique à l'origine de l'inhibition de $\mathrm{M}^{\mathrm{lle}} \mathrm{M}$. soit en lien avec ce cri, cette voix de l'Autre maternel. Dans cette perspective, l'appel d'appel viendrait signer un attachement pulsionnel particulier entre premier et second temps de la pulsion: «Du fond de ma détresse, j'appelle à l'apparition de ta voix comme au premier jour »... 


\section{Conclusion}

La pratique (globalement nouvelle pour les psychologues cliniciens) de l'orientation professionnelle nous ouvre à de nouveaux questionnements. Il n'est pas impossible d'y soutenir une référence psychanalytique, notamment si l'on tient rigoureusement compte de la doctrine des pulsions dans ses liens à l'insertion dans la culture. À ce titre, la pulsion invocante nous permet de penser différemment certains enjeux autour de la recherche de vocation, en repérant cette position subjective de «l'appel d'appel».

Par la prise en compte des représentants langagiers de la pulsion invocante dans le transfert, le clinicien loin de forcément développer la vocation (comme le voudrait le discours social contemporain), peut au contraire favoriser une nouvelle position qui consiste plutôt à renoncer à jouir d'un certain versant de la voix : renoncer à se savoir appelé par l'Autre, car à l'appel du sujet, l'Autre ne répond jamais. Ce passage, $\mathbf{M}^{\text {lle }} \mathrm{M}$. nous l'indique avec ses mots lorsqu'elle passe du «pas assez » (qui relève du trop plein de la voix) au «j'attendais rien» (qui laisse une place pour le non-savoir). En termes plus freudiens, cette nouvelle position, identifiée à un passage par le troisième temps de la pulsion, nous mettrait donc sur la voie d'un renoncement pulsionnel vocal, ainsi que ses effets sur l'insertion sociale $^{6}$.

frédéric vinot

université de nice sophia-antipolis frederic.vinot@yahoo.fr

\section{Notes}

1. On aura reconnu ici une position hystérique qui se développe très sensiblement. L'hystérique, en effet, arbore son insatisfaction et plaide ainsi pour une jouissance suprême, sublime. Ce faisant le phallus, voie offerte à la jouissance de tout un chacun, est considéré non pas comme un signifiant, mais comme un objet qui se révèle insuffisant, incapable d'accomplir ses promesses. C'est ainsi que le «poteau indicateur», figure emblématique des liens entre orientation et fonction phallique, sera pour l'hystérique objet de toutes les attentes, et de tous les dénigrements, une fois édifié. C'est aussi pourquoi l'hystérique maintien cette recherche réitérée d'un maître qui puisse répondre sans ambiguïté à sa question. À la liste habituelle des maîtres (prêtre, médecin, professeur, psychanalyste), on peut rajouter aujourd'hui de façon flagrante le conseiller d'orientation.

2. «Rien ne force personne à jouir, sauf le surmoi. Le surmoi, c'est l'impératif de jouissance — Jouis !».

3. Cette transposition s'appuie notamment sur les travaux d'Alain Didier-Weill (1995) et Jean-Michel Vivès (2002).

4. Il s'agit bien de passivation et non de passivité (Penot, 2001, 14).

5. «Les rêves les mieux interprétés gardent souvent un point obscur; on remarque là un nœud de pensées que l'on ne peut défaire, mais qui n'apporterait rien de plus au contenu du rêve. C'est l'ombilic du rêve, le point où il se rattache à l'Inconnu. Les pensées du rêve que l'on rencontre pendant l'interprétation n'ont en général pas d'aboutissement, elles se ramifient en tous sens dans le réseau enchevêtré de nos pensées. Le désir surgit d'un point plus épais de ce tissu, comme le champignon de son mycélium» (Freud, 1997, 446).

6. Sur cette question du renoncement pulsionnel vocal, nous renvoyons à notre thèse (Vinot, 2006) et d'autres publications en cours. 


\section{4}

Filigrane, automne 2007

\section{Références}

Didier-Weill A., 1995, Les trois temps de la Loi, Paris, Seuil.

Freud S., 1895, Esquisse d'une psychologie scientifique, in Naissance de la psychanalyse, Paris, PUF, 1996.

Freud S., 1900, L'interprétation des rêves, Paris, PUF, 1997.

Freud S., 1914, Pour introduire le narcissisme, in La vie sexuelle, Paris, PUF, 1989.

Freud S., 1915, Pulsions et destins des pulsions, in Métapsychologie, Paris, Gallimard, 1968.

Freud S., 1923, Le moi et le ça, in Euvres complètes, vol. XVI, Paris, PUF 1991.

Guichard J. et Huteau M., 2001, Psychologie de l'orientation, Paris, Dunod.

Homère, L'odyssée, Paris, GF-Flammarion, 1965.

Lacan J., 1964, Le Séminaire Livre XI. Les quatre concepts fondamentaux de la psychanalyse, Paris, Seuil, 1973.

Lacan J., 1962-1963, Le Séminaire Livre X. L'angoisse, Paris, Seuil, 2004.

Lacan J., 1972-1973, Le Séminaire Livre XX. Encore, Paris, Seuil, 1975.

Lecourt E., 1992, Freud et le sonore, Paris, L'Harmattan.

Penot B., 2001, La passion du sujet freudien, Toulouse, Erès.

Vandermersch B. et Chemama R., 1998, Dictionnaire de la psychanalyse, Paris, Larousse.

Vinot F., 2006, Des fondements vocaux de la socialité: insertion de l'objet voix dans la clinique de l'exclusion, thèse de psychologie, soutenue à l'Université de Nice Sophia-Antipolis.

Vinot F., 2007, D’une dimension vociférante du transfert, intervention au colloque « Réalités du traumatisme, Réel du trauma» organisé en novembre 2007 par l'équipe de Psychopathologie Clinique et Psychanalyse de l'U.N.S.A. (actes à paraître).

Vives J.-M., 2002, Les enjeux de la voix en psychanalyse dans et hors la cure, Grenoble, PUG. 